\title{
Electro-bioremediation at the prototype scale: what it should be learned for the scale-up
}

\author{
S. Barba ${ }^{a}$, R. López-Vizcaíno ${ }^{\mathrm{b}}$, C. Saez ${ }^{\mathrm{c}}$, J. Villaseñor $^{\mathrm{a}}$, P. Cañizares ${ }^{\mathrm{c}}$, V. Navarro ${ }^{\mathrm{b}}$, \\ M.A. Rodrigo ${ }^{c}$
}

${ }^{a}$ Chemical Engineering Department. Research Institute for Chemical and Environmental Technology. University of Castilla La Mancha, 13071, Ciudad Real, Spain.

${ }^{\mathrm{b}}$ Geoenvironmental Group, Civil Engineering School, University of Castilla-La Mancha, Avda. Camilo José Cela s/n, 13071 Ciudad Real, Spain.

${ }^{c}$ Chemical Engineering Department. Faculty of Chemical Sciences and Technology. University of Castilla La Mancha, 13071, Ciudad Real, Spain.

\begin{abstract}
A large scale electro-bioremediation test is carried out in a prototype of $32 \mathrm{~m}^{3}$. Soil polluted with a mixture of two herbicides 2,4-D and oxyfluorfen undergoes a combined treatment in which the electrochemically assisted remediation produced by six electrodes placed in an hexagonal alternating configuration is aimed to be enhanced with the addition of concentrated activated sludge in a central position of the prototype. Results demonstrate that this technology can remove pollutants down to a very low concentration although, as compared to a previous study, its performance does not differ significantly of that followed in a process in which bioremediation was not included. In both studies, volatilization was found to be the key mechanisms to explain the removal of both herbicides. After a treatment period slightly over month, only $7.10 \%$ of the $2,4-$ $\mathrm{D}$ and $9.22 \%$ of the oxyfluorfen remained in soil and these values are very similar to those obtained by applying non biological EKF in which 10.10 of the 2,4-D and $13.03 \%$
\end{abstract}


of the oxyfluorfen remained in the soil after the treatment. Results pointed out that it is very difficult to optimize simultaneously electrokinetic and biological processes because most of the processes caused by the electric field affect negatively to the microorganisms viability. Results also pointed out the significance of the sampling procedure to characterize an electro-bioremediation process at full scale.

\section{Keywords}

Scale-up; full scale; soil remediation; electro-bioremediation; electrokinetic

\section{Highlights}

- Electro-bioremediation can efficiently deplete the concentration of herbicides

- Increase in temperature caused by ohmic loses is the primary removal mechanism

- Electrokinetic processes affect negatively to the microorganisms viability

- Sampling procedure is a key to characterize an electro-bioremediation process 


\section{Introduction}

The occurrence of soil polluted with pesticides is a dangerous environmental issue that may also produce the contamination of groundwater due to the infiltration of these compounds [1]. This pollution entails a highly negative impact to the environment, preventing the use of water reservoirs in urban supplies. It also becomes a risky situation for animals, plants and many other different organisms, which could be in contact with the polluted soil. In addition, and associated to their complex chemical composition, many of the pesticides currently in use are now considered as endocrine disrupters. This means that they can produce serious alterations in the endocrine system, which in the worst case may cause death [2]. Furthermore, pesticide residues remain in the environment for variable times, which often may be very long, extending their negative impact for lengthy periods. For this reason, and because the soil is a natural resource than cannot be renewed, remediation of polluted soil should be mandatory and there is an increasing regulation in most developed countries [3].

There are many conventional technologies available for the remediation of polluted soils, based on chemical, biological, physicochemical or thermal processes $[4,5]$. These technologies may be classified into two groups, in-situ and ex-situ techniques. In-situ technologies focus on the remediation of polluted soil without removing the soil from its original site and they typically exhibit a lower impact and economical cost.

Among these in-situ remediation processes, it can be found the electrokinetic (EK) treatments. These technologies are focused on the remediation of soils with low permeability such as clayey soil, for which conventional techniques are not effective to move the underground water retained [6-10]. Electrokinetic processes consist of 
applying an electric field between couples of electrodes inserted in the soil. Then, electrokinetic phenomena (specifically electro-osmosis, electromigration and electrophoresis) produce the transport of charged species contained in the soil, including pollutants, ions, microorganisms and nutrients [3, 6]. In addition, the electric field exerted produces electrochemical processes, being the most important the electrolysis of water, which generates an acid and basic front caused by the oxidation and reduction of water on the anodic and cathodic surface, respectively. There are many strategies to remediate soil and they depend on the particular placement of electrodes and operation conditions, being the electrokinetic soil flushing and the electrokinetic soil fence two of the most important processes $[11,12]$.

It has been proposed that combining EK treatments with other conventional techniques is a good option to improve the efficiency of pollutant removal and obtain the main advantages of both technologies [3]. Recently, interest has increased in electrobioremediation (EBR), which consists of coupling EK technology with bioremediation. Then, the EK transport processes are combined with biodegradation caused by a microbial culture, and this combination entails an advantage allowing the enhanced insitu removal of pollutants from the soil [13]. There are two different bioremediation strategies and both can be combined with electrokinetic processes [14-16]. One of them consists of adding nutrients in soils to favour the metabolism of the autochthonous soil microbial population (biostimulation). The other option consists of using an acclimatized microbial culture for the pollutant degradation (bioaugmentation). It includes the possibility of employing reactive permeable biobarriers, in which the pollutant is moved all over the soil and it has to pass through the biobarrier inserted, normally, in the central position of the soil area that aims to be remediated [17]. Despite the good prospect of EK treatments to remediate soils with low permeability, 
combination of EK and bioremediation processes has some well-known limitations such as the extreme $\mathrm{pH}$ and the high temperatures that can be produced, which are two of the most critical parameters to keep the microorganisms active [16, 18]. Anyhow, these drawbacks could be easily overcome, as it was pointed out in a previous work in bench scale set-up $\left(2.4 \mathrm{dm}^{3}\right)$, in which oxyfluorfen polluted soil underwent electrobioremediation [19]. Now, the interest of the authors is to apply this technology in a prototype-scale facility $\left(32 \mathrm{~m}^{3}\right)$, using a 2,4-D and oxyfluorfen clayey real polluted soil, in order to know if electro-bioremediation technique works as in bench scale and to identify the technology limitations, in the case that they appear. To do this, a case study consisting of remediating a simulated spill of both herbicides is faced. In previous works, it was found that size of the experimental facility is a very important input because although the process occurring in the system are the same, the results obtained may be completely different [20-22]. Hence, this application of EBR technology to remediate in-situ a herbicide polluted soil in real scale becomes the main novelty point of this work as compared to previous existing works.

\section{Materials \& Methods}

Soil. The soil employed in this research is a low-plasticity clay (CL) according to the Unified Soil Classification System [23, 24] and it comes from a zone with important agrarian activities near to Toledo (Spain). Its main characteristics can be found in previous works of our group [25-33].

Herbicides. Two different commercial solutions have been selected to simulate an accidental herbicide spill. Esteron 60, a commercial herbicide with a $60 \%$ (p/v) of 2,4dichlorophenoxyacetic acid (2,4-D) has been used as model of polar herbicide. This 
herbicide has been provided by Dow AgroSciencesIbérica (Madrid, Spain). Oxyfluorfen has been selected as model of nonpolar herbicide. The source of this substance was a commercial emulsion of Fluoxil 24 from Cheminova Agro S.A. (Madrid, Spain). It is composed by $24 \%(\mathrm{p} / \mathrm{v})$ of oxyfluorfen dissolved in aqueous solution by the emulsifier action of xylene (59\%), cyclohexanone (13\%) and calcium dodecylbenzenesulfonate $(4 \%)$.

Soil remediation prototype. The EBR test was conducted in a prototype located inside the Institute of Chemical and Environmental Technologies of UCLM (Ciudad Real, Spain). The prototype is composed by an electrokinetic soil remediation reactor with soil-treatment capacity of $32 \mathrm{~m}^{3}$ and the dimensions were $2 \mathrm{~m}$ of height and a square plant of $16 \mathrm{~m}^{2}(4 \mathrm{~m} \times 4 \mathrm{~m})$. The soil was disposed into the reactor in layers. A compaction process was conducted in each layer to obtain a soil with hydro-mechanical properties similar to those of a real soil in the environment. A detailed description of the reactor construction process and the steps of the soil preparation were explained in a previous work focused on scale-up of electrokinetic remediation processes [25]. To arrange the electro-bioremediation process, the electrodes (cylinders of graphite; diameter=15 $\mathrm{cm}$ and length=100 $\mathrm{cm}$ ) were positioned in semipermeable electrolyte wells (PVC cylinders with lateral perforations; diameter=31.5 $\mathrm{cm}$ and length=140 cm) distributed in hexagonal form. This electrode configuration corresponds to an electrokinetic fence composed on sequence of six alternating electrodes (three cathodes and three anodes). To monitor the different parameters during the test, thermocouples and water pressure sensors (or "divers") were located at different positions into the soil. Moreover, a complete distribution of twenty micro-boreholes (PVC cylinders with lateral perforations; diameter $=4.0 \mathrm{~cm}$ and length $=110 \mathrm{~cm}$ ) were disposed into the soil 
for liquid sampling and to monitor the $\mathrm{pH}$, electrical conductivity and temperature of the porewater (Fig. 1).
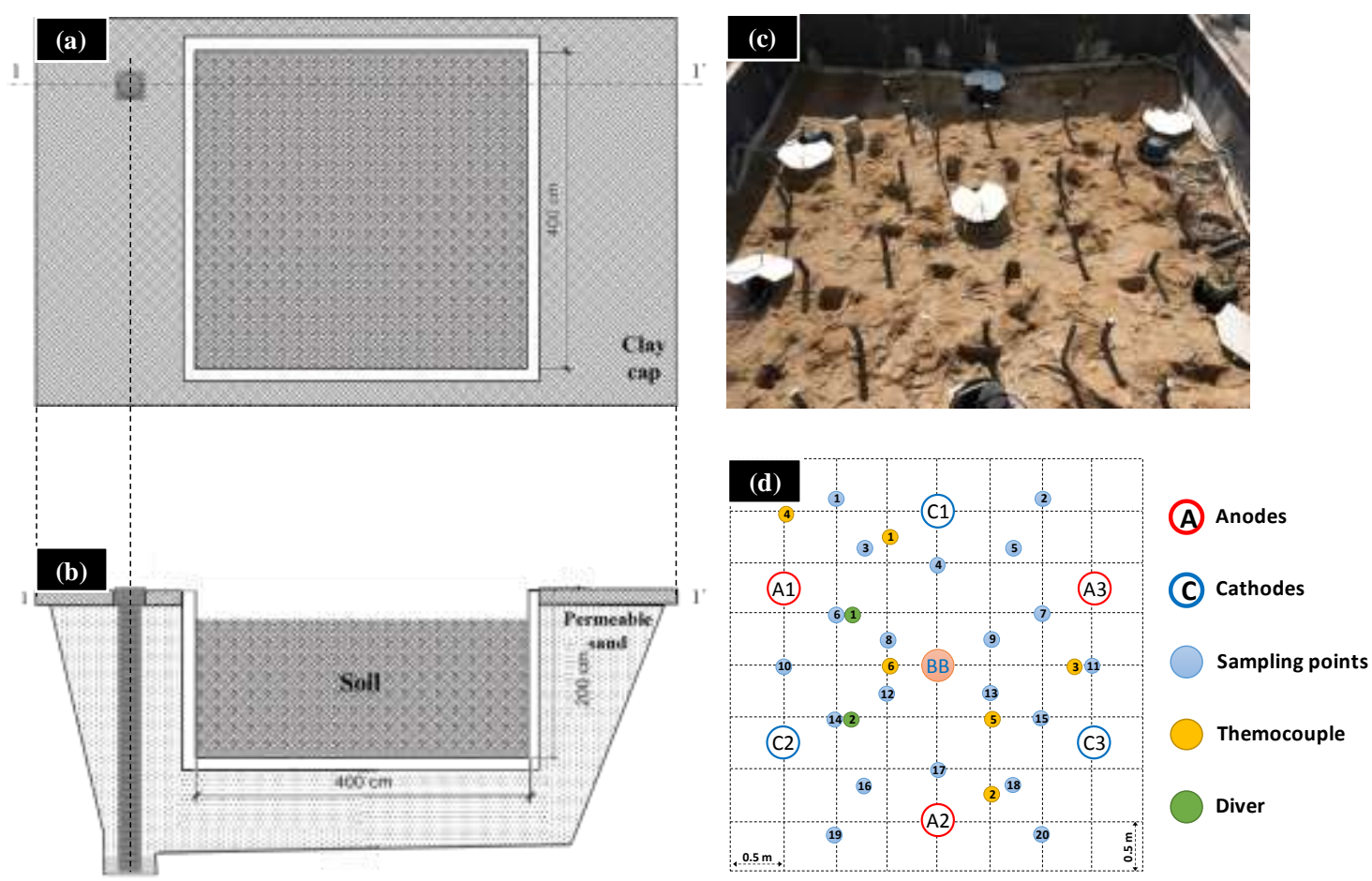

Figure 1. (a) Plan view, (b) 1-1'section, (c) picture of EBR prototype and (d) scheme of the location of electrode wells and the main instrumentation (BB, biobarrier).

Start-up procedure. The EBR test was carried out after finishing a previous EKR test in which Electrokinetic Fence (EKF) technology was evaluated [26, 27]. The central well of previous EKF test (design to make the initial herbicide discharge) was replaced by a new well designed to house a biobarrier (see BB in Figure 1). In EBR test, the accidental herbicide spill was discharged in the 7 wells (6 electrolyte wells +1 BB well) in order to achieve a better initial distribution of the herbicides throughout the soil. The initial concentration of 2,4-D and Oxyfluorfen was 5.991 and $4.742 \mathrm{mg} \mathrm{kg}_{\text {dry soil }}^{-1}$. The biological barrier placed in the soil was a microbial consortium adapted for 2,4-D and oxyfluorfen degradation. This microbial consortium was obtained from an activated sludge and fed for a week, before their addition in the soil, with a concentration of 300 
ppm of glucose, $70 \mathrm{ppm}$ of 2,4-D and $70 \mathrm{ppm}$ of oxyfluorfen as carbon source in Bushnell-Hash broth, a culture media adequate to herbicide-degrading microbial consortium. Then, the microorganisms were introduced in the soil through the central well mixing them with sand which acts as a support of these microorganisms. The test started when the power supply, MAGNA POWER ELECTRONICS (7.5 Kw: 0-1000 V and 0-25 A) was turned on with a constant voltage of $168 \mathrm{~V}\left(1.0 \mathrm{~V} \mathrm{~cm}^{-1}\right)$.

Analytical techniques. The water content and dry density of the soil were measured following the standard procedures $[34,35]$. Soil temperature and water table were measured with conventional thermocouples PT-100, and divers sensors from Schlumberger (Netherlands). The characteristic parameters of the porewater $(\mathrm{pH}$, conductivity and temperature) were monitored with a multiparametric analyzer sensION+ MM150 DL from Hach (Colorado, USA).

The herbicide in solid and liquid samples concentration was analyzed by HPLC model Agilent 1100 with an UV detector, from Agilent Technologies (California, USA) following the analytical procedures described in previous works of our group which can be found elsewhere [28-33].

Moreover, soil microorganisms concentration was expressed as Colony Forming Units (CFU) per gram of dry soil [36]. Samples were prepared getting $1 \mathrm{~g}$ of wet soil and adding $10 \mathrm{ml}$ of $\mathrm{NaCl}$ solution $(0.9 \%)$ and it is mixed during 3 min with a vortex agitator. Then, it was taken the supernatant $(100 \mu \mathrm{L})$ and dispel on Petri dishes which contains $\mathrm{LB}$ as solid media culture $\left(10.0 \mathrm{~g} \mathrm{~L}^{-1} \mathrm{NaCl}, 5.0 \mathrm{~g} \mathrm{~L}^{-1}\right.$ yeast extract and $10.0 \mathrm{gL}^{-1}$ casein peptone, $15 \mathrm{~g} \mathrm{~L}^{-1}$ of European Bacteriological Agar and $10.0 \mathrm{~g} \mathrm{~L}^{-1}$ of glucose as carbon source). The plates were incubated for $24 \mathrm{~h}$ at $26.5^{\circ} \mathrm{C}$. 


\section{Results \& Discussion}

Figure 2 shows the changes in the current produced during the 1-month EBR test. During this period, the electric field was targeted to be kept at $1.0 \mathrm{~V} \mathrm{~cm}^{-1}$ between each pair of consecutive electrodes that conforms the hexagonal alternating electrode configuration used. As observed, the total intensity produced fluctuates around an average value of $14 \mathrm{~A}$, with maximum values around 18 and minimum values around 6 A. These changes can be explained in terms of the partial dewatering of soil over time, which made necessary the addition of water to the electrode wells and which lead to increases in the ohmic resistance of the soil. In addition, despite being controlled, cell voltage shows decreases over time, which can be explained in terms of the punctual switching off of the experimental setup in order to do maintenance work and sampling.

(a)

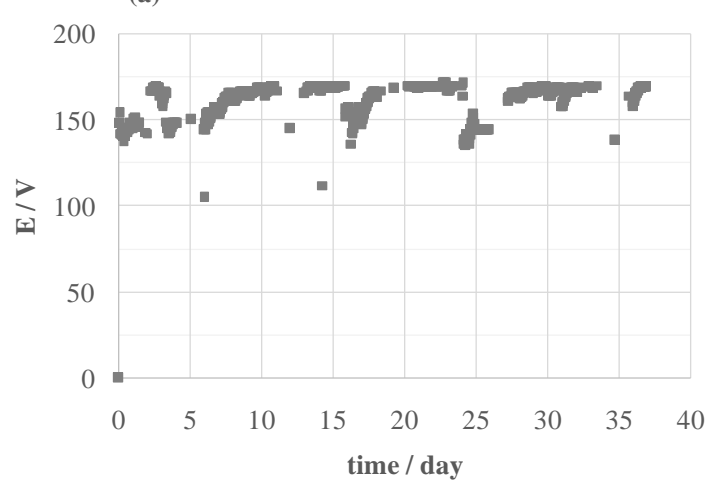

(b)

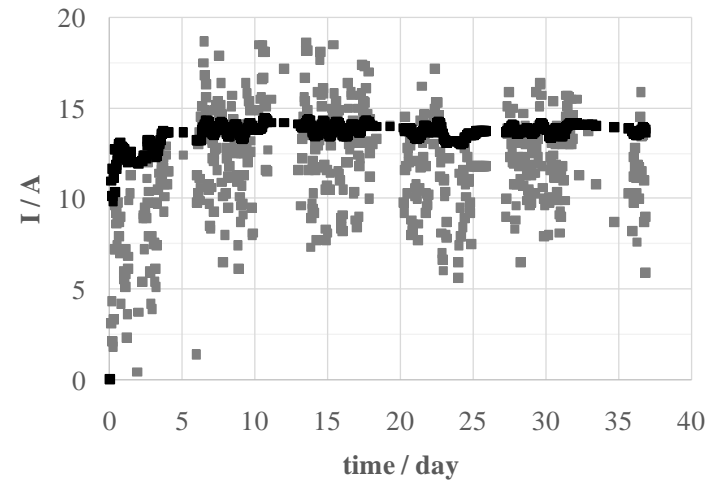

Figure 2. (a) Changes in the voltage and (b) current intensity produced during the operation of the EBR process. Grey markers, experimental values; black markers, average values.

The intensity produced by the electric field can be considered as the driving force of the electroremediation processes and, hence, it should be directly associated to the changes observed in the electrolyte wells and, in turn, in the soil. These changes are not only related to reactivity of species but also to their transport and the heat transfer to 
environment and they should be reflected on the values of three important parameters: $\mathrm{pH}$, conductivity and temperature. The changes of these three parameters in the electrodes wells are shown in Figure 3 and in the soil in Figure 4.

As it can be seen, $\mathrm{pH}$ in the three anodic wells turns into strongly acidic, while it becomes strongly alkaline in the cathodic wells. These changes are caused by the anodic oxidation and cathodic reduction of water and hence they are indicative of the electrochemical reactivity of the system. From the transient point of view, the changes are very rapid and in less than 100 hours the final values are reached. From this time on, they are kept until the end of the test. No significant discrepancies are found between the values reached in each of the three anode wells and between these values in the three cathode wells.

Dynamic response of the conductivity is much slower and the plateau in the conductivity vs time curve is only seen after 700 hours of operation of the prototype. Over the test, conductivity increases in both the anodic and cathodic wells, although the change in more pronounced in the cathodic wells where, in addition, there are more discrepancies between the final values reached in each of the individual wells. These changes in the conductivity cannot only be explained in terms of the production of protons and hydroxyl ions during the oxidation and reduction of water but most importantly because of the transport of ions contained in the soil towards the electrodes wells: cations to the cathode and anions to the anode. At this point, it is worth to point out that electro-osmotic fluxes were negligible (no water was collected in the cathodes and in fact some water had to be added in order to avoid the dryness in these wells). Hence this transport should be associated almost exclusively to electromigration.

Temperature also increases during the test as a consequence of the ohmic heating of the soil. Other processes influence on the values of temperature such as loses of heat to 
environment (by convention) and the evaporative cooling. As a result, temperature also reaches a steady state value. The time constant of this heat-transfer controlled process is between that of the $\mathrm{pH}$ (reactivity controlled) and conductivity (mass transport controlled). In addition, it should be pointed out that changes are more important in cathodes than in anodes, despite of the higher conductivity of the liquid contained in the cathodic electrode wells, which initially should be reflected in lower ohmic loses. This can be explained in terms of the layer of carbonates that it is formed on the surface of the cathodes and that increases importantly the electric resistance of electrodes during the operation period.
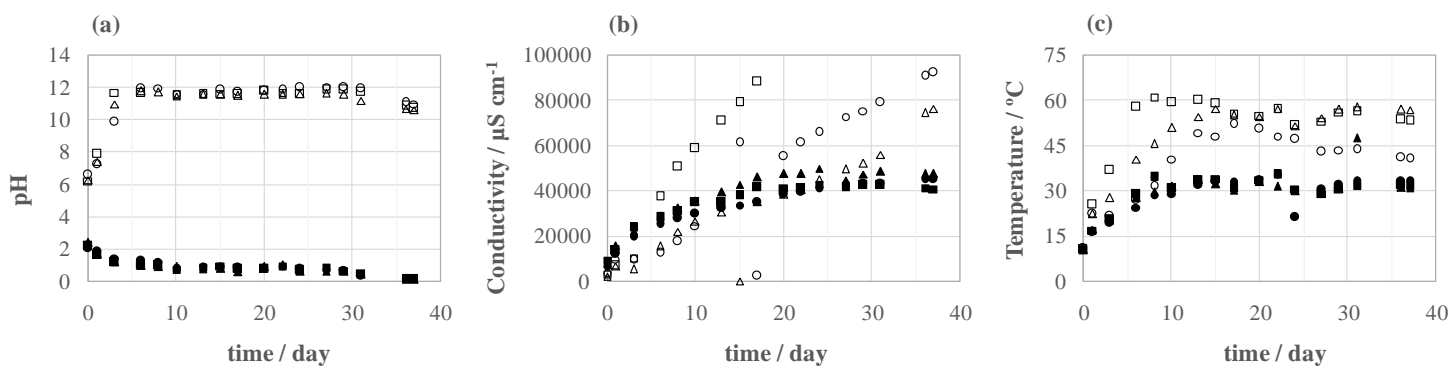

Figure 3. Changes in the (a) $\mathrm{pH}$, (b) conductivity and (c)temperature of the electrolyte contained in the electrode wells. Cathodes: empty marker; Anodes: full marker. Well position: $\circ 1, \Delta 2, \square 3$ (see Figure 1).

Changes observed in the three anodic and three cathodic wells are reflected in the soil. Thus, it can be seen how the wells become the central points from which the changes in soil are produced (Figure 4). It is important to point out that although these changes are very important, there is a central zone in which they are less relevant. In the case of the $\mathrm{pH}$, neutralization of the acidic and basic fronts keep this central zone in values suitable for microbial life. In addition, it can be seen how the faster and more important transport of the acidic front helps to neutralize the nearness of the cathodes at the end of the test. Regarding conductivity, it is seen how there is an exhaustion of salts from the 
central point and an accumulation in the electrodes wells, which is very important in the case of the cathodes. This can be negative for the viability of the biological cultures, because they need nutrients to metabolize the organic pollution and to survive. Particularly, just in the points were the $\mathrm{pH}$ is compatible with life, the conductivity seems to become negligible and hence the amount of ionic nutrients may become insufficient to preserve life. The very high values reached in the wells is also negative for life because microorganisms cannot develop satisfactorily in highly-saline media. In addition, in this case, this negative prospect can be linked to the much more negative effect on microorganism viability of the $\mathrm{pH}$ and, hence, it is not foreseeable that microbial life will be important in this zone. Regarding changes in the temperature of the soil, it has to be pointed out that they are rather important. The most outstanding point is that they are much higher in the cathode proximities and this can be explained in terms of the higher temperature reached in the wells. In turn, this higher temperature can be easily associated (as commented before) to the increase in the electric resistance caused by the deposit of carbonates on the surface of the cathodes.

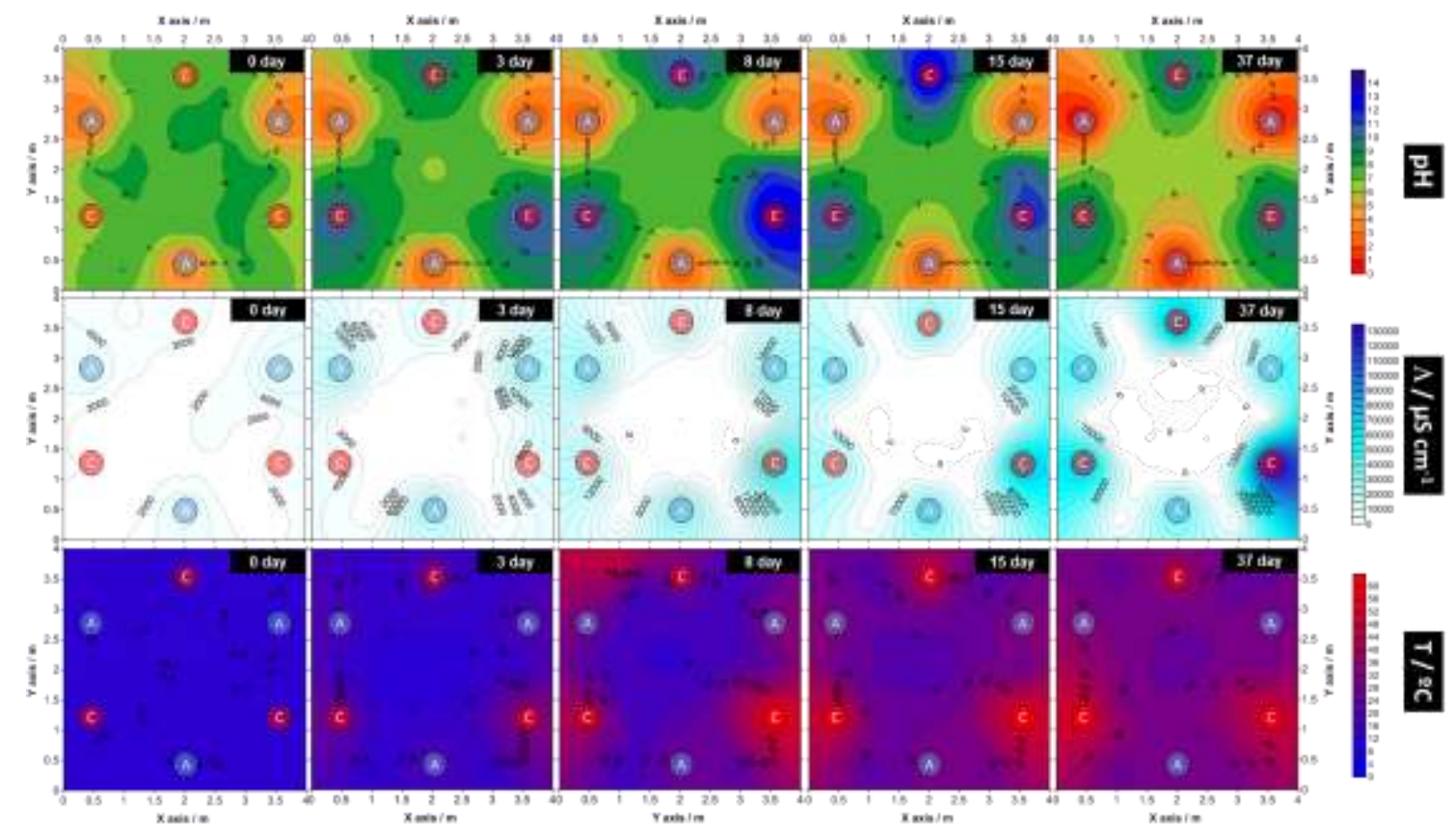


Figure 4. 2-D maps showing changes over time of $\mathrm{pH}$, conductivity and temperature in the soil remediated.

Hence, according to these three parameters, the most relevant negative influence of transport processes induced by the electric field applied is focused on the concentration of nutrients, which can become a limiting factor of the microorganism's growth, as the conductivity tendency seems to point out. In order to know more about this point, the changes in the concentrations of the main cations and anions were monitored. As expected, monovalent chloride and nitrates are rapidly transported to the anodes and exhausted from soil (Figure 5). This can become a significant problem as nitrates have an important role in the metabolism of microorganisms not only as nutrients but also as electron acceptors (helping aerobic denitrification bacteria to remove the organic pollution).

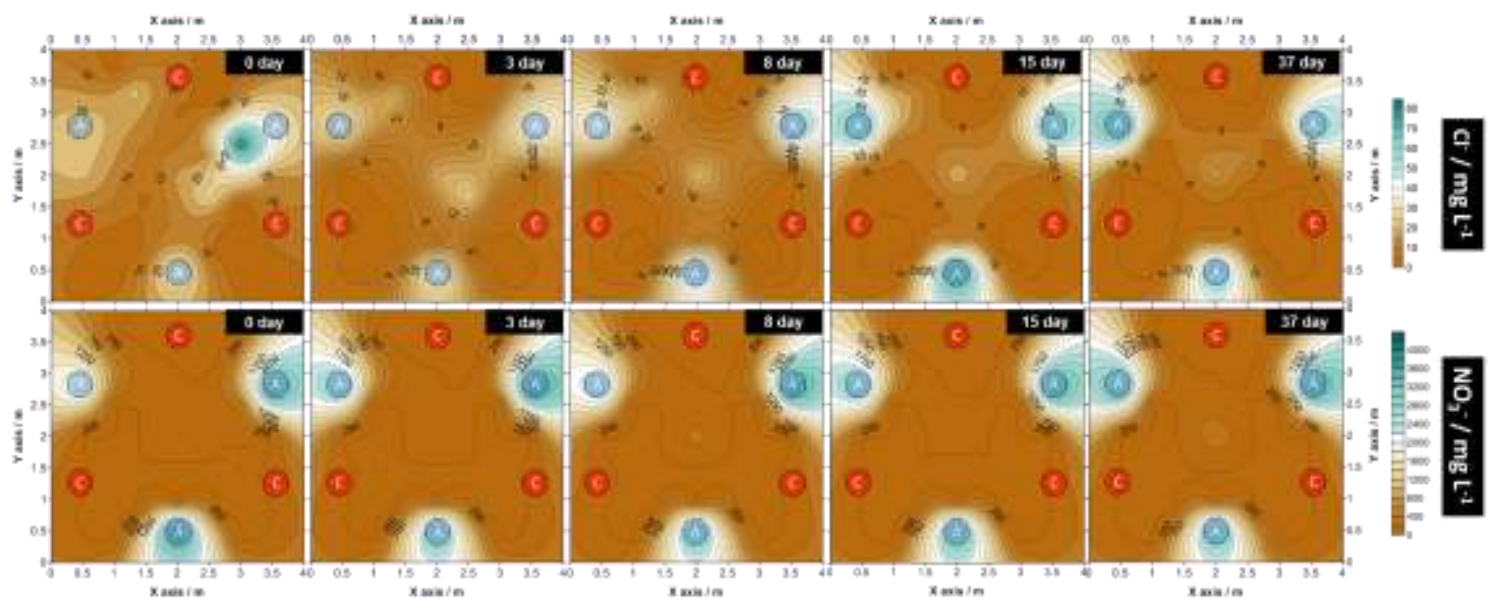

Figure 5. 2-D maps showing changes over time of chloride and nitrate anions in the soil remediated.

Same occurs with sulfate anions, which are rapidly concentrated in the cathode and the same observation also applies for phosphate, although in a less important way (Figure 6). In this case, it is important to point out that we are measuring the phosphate in the liquid and that this compound is expected to be precipitated in the soil. For this reason, 
it seems to disappear. In fact, it does not disappear but it is simply fixed in the soil by precipitation and it is not available for being dragged with water.

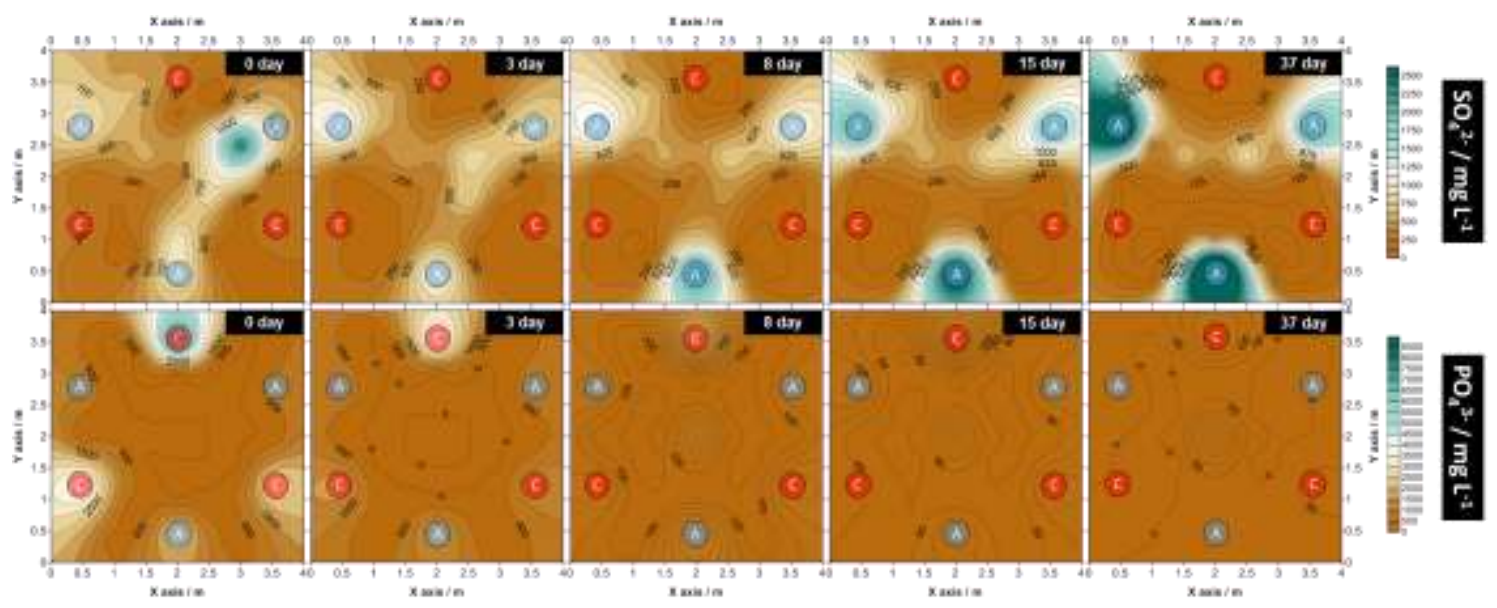

Figure 6. 2-D maps showing changes over time of sulfate and phosphate anions in the soil remediated.

The transport of cations is in the opposite direction of that of anions (Figure 7). They are concentrated in the nearness of the cathodes, where the alkaline $\mathrm{pH}$ contributes to the fixation of calcium precipitates. In addition, the removal of ammonium can be explained in terms of the formation of ammonia in the electrodes and the stripping with the hydrogen produced during water reduction. It is particularly relevant the exhaustion of ammonium after only one week. In this case, the alkaline $\mathrm{pH}$ reached in the cathodic wells may promote the transformation of ammonium ion into ammonia, which is stripped from these wells, explaining its rapid withdrawal.

Hence, electromigration is a very important problem: anions and cations management seems to be a major handicap in the combination of bio and electroremediation processes. In particular, it is especially relevant that the three most important nutrients required for life (nitrate, ammonium and phosphate) are depleted during the treatment, despite they were initially present at a very high concentration and this can have a negative influence on biological results. 


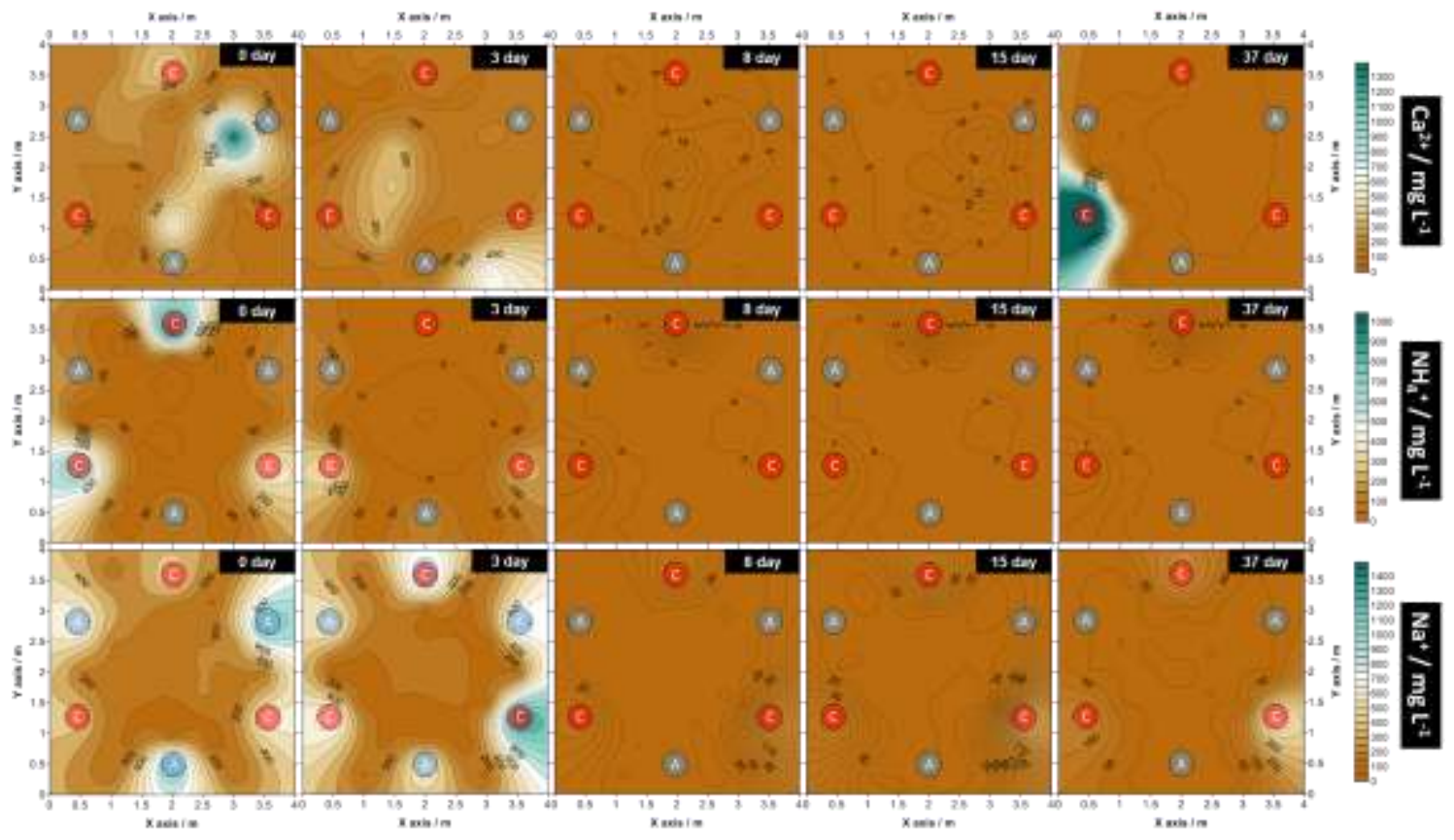

Figure 7. 2-D maps showing changes over time of calcium, ammonium and sodium cations in the soil remediated.

Figure 8 focuses on the amount of compounds collected in the electrodes. As it can be seen the concentration is very small, almost negligible. This points out the low significance of the electrokinetic processes in the transport of organics when the soil undergoes an electric field between electrodes. This low significance was pointed out in a previous work in which the electrokinetic soil fence technology was applied in the same prototype $[21,22]$. In this case concentrations are really negligible and below the $0.31 \%$ and $0.28 \%$ of $2,4-\mathrm{D}$ and oxyfluorfen collected in that work. This can be explained by the lower initial concentration of both pollutants in the soil. Thus in the previous experiments the concentration of 2,4-D and oxyfluorfen were $20.11 \mathrm{mg} \mathrm{kg}^{-1}$, while in the present work they are only of 4.74 and $5.99 \mathrm{mg} \mathrm{kg}^{-1}$, respectively. 

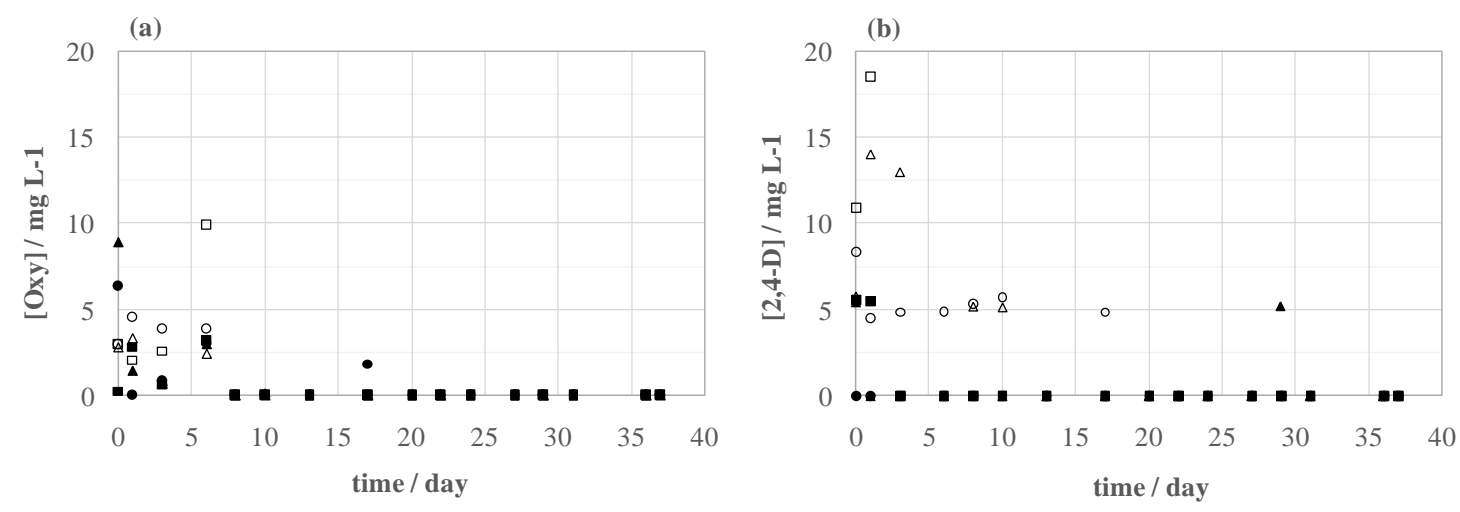

Figure 8. Changes in the concentration of oxyfluorfen and 2,4-D in the electrolyte contained in the electrode wells. Cathodes: empty marker; Anodes: full marker. Well position: $\circ 1, \Delta 2, \square 3$ (see Figure 1).

However, the most important piece of information that can be extracted from this work comes from the changes in the concentration of microorganism and pollutants over the remediation test. This information, taken from the micro-boreholes disposed into the soil for liquid sampling, is shown in Figure 9. As it can be observed, microorganisms are progressively destroyed during the treatment and eventually, they are only found in the central region of the prototype, where there are mild conditions in temperature and $\mathrm{pH}$ and just where the main handicap is the low concentration of nutrients to perform properly their metabolism. According to these results, the total amount of microorganism is three $\log$ units lower after the test (it decreases from 7.05 down to 4.77 in $\left.\log _{10}\left(\mathrm{CFU} \mathrm{dm}{ }^{-3}\right)\right)$.

Regarding the concentration of the two herbicides, it decreases over time, down to very low values showing, in addition, a final 2-D map in which the distribution of concentrations is very different from that of the initial map. This concentration seems to be lower near the electrodes and higher in the central point of the prototype, just the point in which the concentration of microorganism seems to be higher and where temperature is much lower. This suggests that biological remediation is not an important 
mechanism in EBR test carried out, at least not as important as the evaporation of the pollutants. At this point it is worth to point out that evaporation was found to be the most important mechanisms in the EKF tests carried out in the previous work and that also in the present work the maps of temperature and herbicide concentration almost match indicating a higher removal in the points with higher temperature.

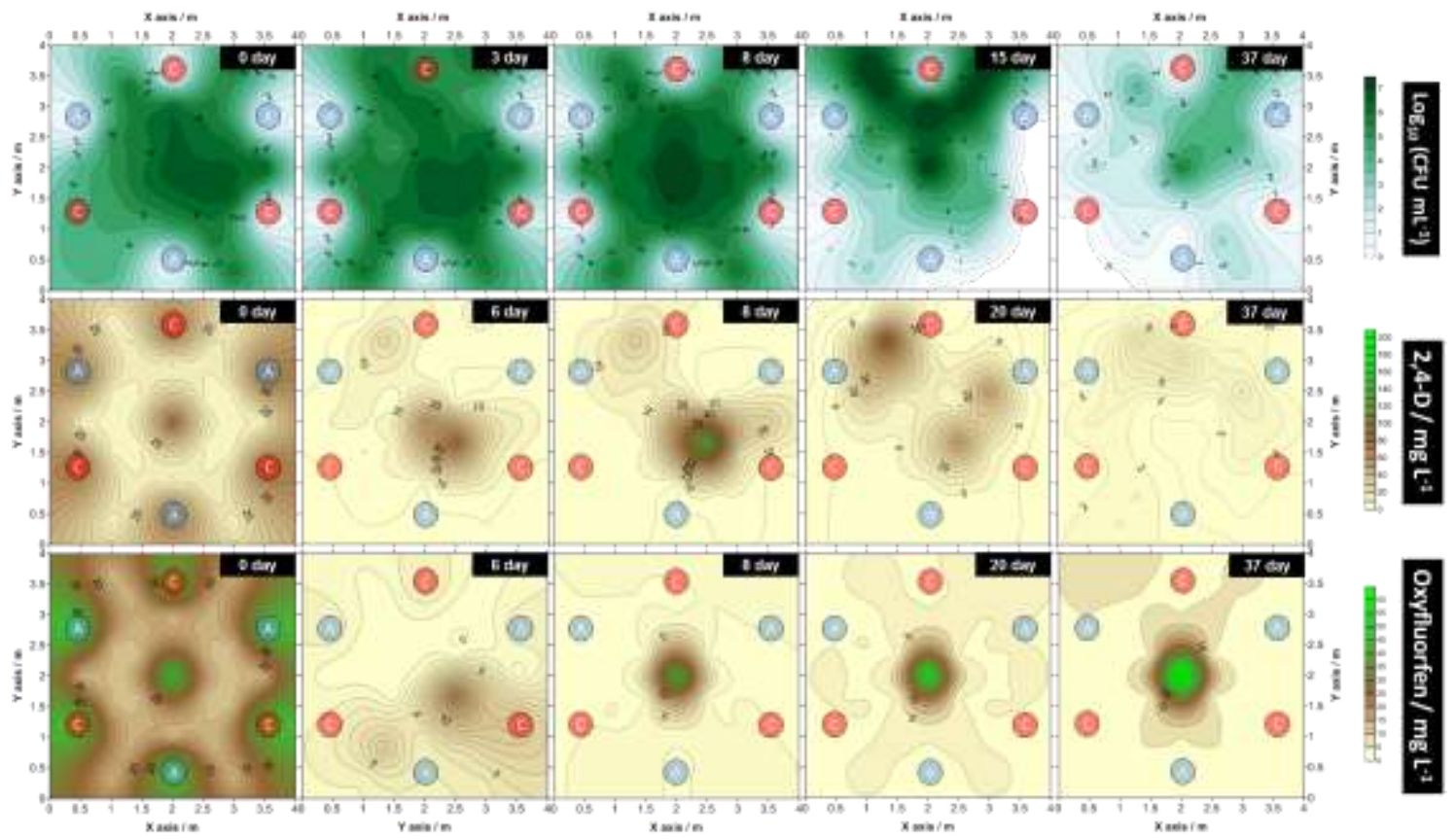

Figure 9. 2-D maps showing changes over time of microorganisms, oxyfluorfen and 2,4-D in the soil remediated.

Anyhow, the decrease in the concentration of both pollutants is very important and the final concentrations reached are very similar. Thus, in Figure 10 it is plotted the variation over time of the average concentration of herbicide contained in the soil according to the sampling points. A continuous decrease trend is observed, showing that the concentration decreases in more than 5 times in a period only slightly over 1 month. In fact, after the treatment period only $7.10 \%$ of the $2,4-\mathrm{D}$ and $9.22 \%$ of the oxyfluorfen remain in soil. These values are very similar to those obtained in the 
previous work focused on EKF $[21,22]$ in which 10.10 of the 2,4-D and $13.03 \%$ of the oxyfluorfen remained in soil after the treatment.

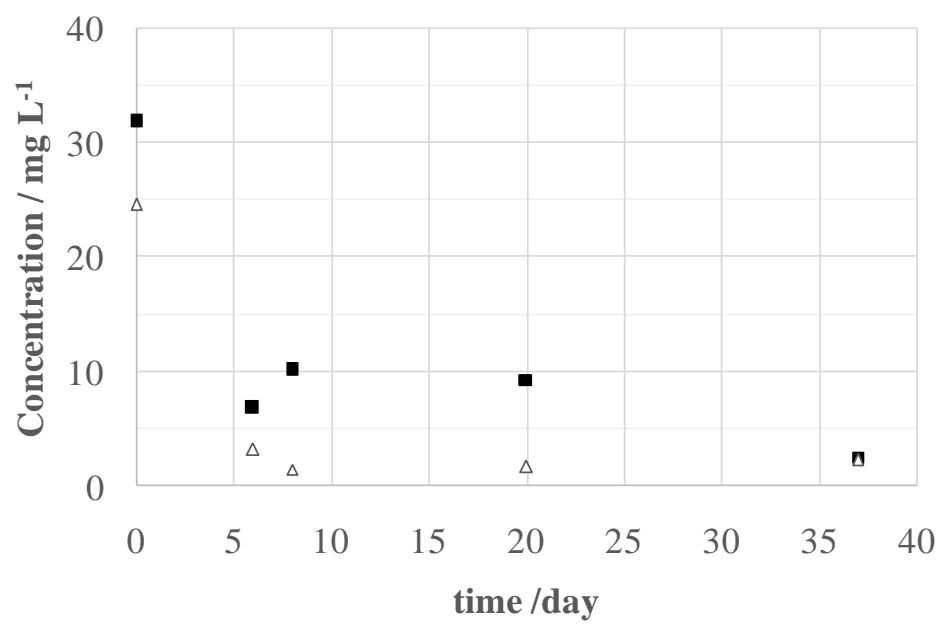

Figure 10. Changes in the average concentration of oxyfluorfen (unfilled markers) and 2,4-D (filled markers) contained in the soil during the remediation test.

A last very important point in this work is the comparison of the values of the most important parameters evaluated from the liquid sampling (collected in the microboreholes disposed into the soil) and those that include the postmortem characterization of the soil contained in the prototype. At this point, it is important to take in mind that the postmortem analysis is destructive and, hence, it cannot be applied during the operation of the electroremediation process, because it can interfere in the results. However, the maps obtained in this characterization can be considered as more realistic and they reflect truly the result of the test, (sampling of micro-boreholes only consider the free porewater while postmortem analysis takes into account interstitial porewater and soil)

Figure 11 shows the values obtained in this characterization. No significant differences are found between $\mathrm{pH}$ and conductivity. However, these differences become more important in the concentration of alive microorganisms and pollutant concentration. In 
the case of the microorganism concentration, the postmortem characterization indicates that the population of microorganisms that survive to the application of the electric field is higher than those that the monitoring wells describe. Thus, the initial log concentration of microorganisms was 6.39 (expressed in $\log _{10}\left(\mathrm{CFU} \mathrm{kg} \mathrm{kg}^{-1}\right)$ ). Both measuring systems indicates an important decrease, which is lower in the center of the prototype but sampling in the liquid indicates a decrease much higher as the logarithmic concentration decreases to 3.21 (around three log units), while the postmortem analysis indicates that this decrease is only down to 5.17 (slightly over one log unit).
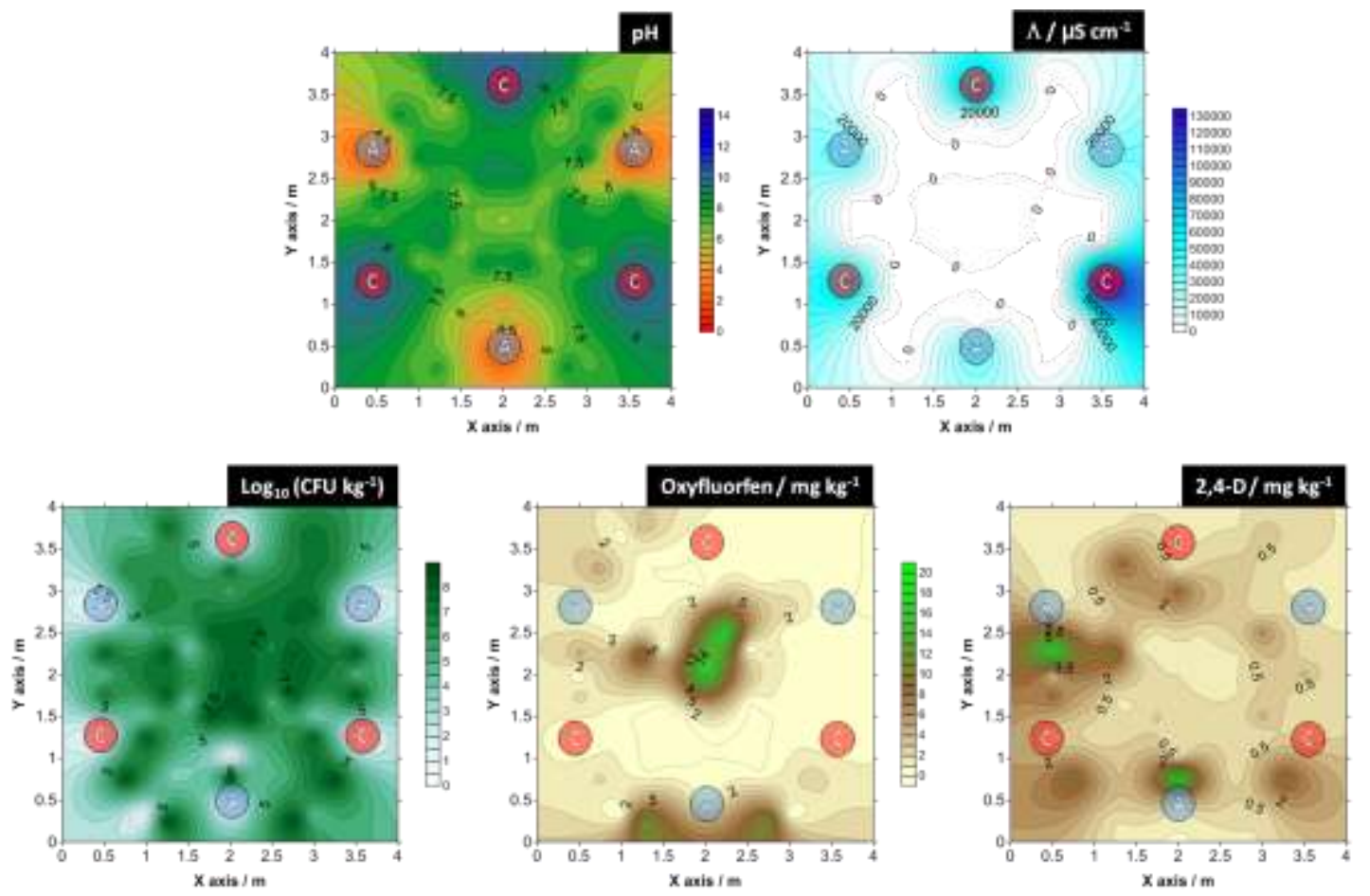

$2,4-\mathrm{D}^{\mathrm{mg}} \mathrm{kg}$

Figure 11. 2-D maps obtained by postmortem characterization of, $\mathrm{pH}$, conductivity, microorganisms, oxyfluorfen and 2-4-D in the soil remediated.

Differences in the pollutant concentrations monitored in the liquid samples and in the soil postmortem analysis are lower. However, they are still very significant, in particular in the micellar pollutant. This indicates (together with the observation made with 
microorganisms) that the transport of particles is less favored than that of soluble species, that is, that soil retains particles that are not contained in the liquid collected in the sampling wells. Thus the total amount of oxyfluorfen decreases from 4.74 to 2.14 according to the soil postmortem test while it decreases down to $0.50 \mathrm{mg} \mathrm{kg}^{-1}$ according to the liquid sampling points clearly indicating the retention in the soil matrix of the particles (as it should happen with the microorganism). In the case of the 2-4-D, the lower differences found between the decrease observed in the liquid sampling (from 5.99 to $\left.0.56 \mathrm{mg} \mathrm{kg}^{-1}\right)$ and the value obtained in the soil postmortem $\left(0.98 \mathrm{mg} \mathrm{kg}^{-1}\right)$ helps to support this explanation.

\section{Conclusions}

From this work, the following conclusions can be drawn:

- electro-bioremediation of soil polluted with oxyfluorfen and 2,4-D behaves as an efficient treatment in terms of removal of both pollutants from soil. However, attending to the mobility of species and the evolution in the concentration of the different parameters that affects bioremediation, the good efficiencies reached does not seem to be a consequence of the electrokinetic or the biological processes but of the volatilization of pollutants caused by the increase in the temperature, which in turn, is due to the ohmic loses. After a treatment period of 35 days, only $7.10 \%$ of the $2,4-\mathrm{D}$ and $9.22 \%$ of the oxyfluorfen remained in soil and these values are very similar to those obtained by applying non biological EKF in which 10.10 of the 2,4-D and $13.03 \%$ of the oxyfluorfen remained in the soil after the treatment.

- it is not easy to optimize simultaneously electrokinetic and biological processes because most of the processes caused by the electric field affect negatively to the 
microorganisms viability. Thus, in the nearness of the electrodes there is a huge increase in the temperature, extreme values of $\mathrm{pH}$ and conductivity and in the central region surrounded by electrodes there is a depletion of nutrients. As a consequence, the population of microorganisms decreases very importantly over the electrobioremediation test.

- the sampling procedure is very important to characterize an electro-bioremediation process at full scale. Despite liquid samples taken at boreholes can be used to monitor changes in the soil during the remediation, the results obtained are not as accurate as those obtained by a postmortem analysis. This is particularly relevant in the description of particulate species such as microorganisms or micelles.

\section{Acknowledgements}

Financial support from the Spanish Ministry of Economy, Industry and Competitiveness and European Union through project CTM2016-76197-R (AEI/FEDER, UE) is gratefully acknowledged. The FPI grant BES-2014-069662 is also acknowledged.

\section{References}

[1] A. Chowdhury, S. Pradhan, M. Saha, N. Sanyal, Impact of pesticides on soil microbiological parameters and possible bioremediation strategies, Indian Journal of Microbiology, 48 (2008) 114-127.

[2] S. Jilani, M. Altaf Khan, Isolation, characterization and growth response of pesticides degrading bacteria, J. Biol. Sci, 4 (2004) 15-20.

[3] M. Rodrigo, N. Oturan, M. Oturan, Electrochemically assisted remediation of pesticides in soils and water: a review, Chemical reviews, 114 (2014) 8720-8745.

[4] J. Virkutyte, M. Sillanpaa, P. Latostenmaa, Electrokinetic soil remediation - critical overview, Science of the Total Environment, 289 (2002) 97-121.

[5] M.A. Rodrigo, N. Oturan, M.A. Oturan, Electrochemically Assisted Remediation of Pesticides in Soils and Water: A Review, Chemical Reviews, 114 (2014) 8720-8745.

[6] K.R. Reddy, C. Cameselle, Electrochemical remediation technologies for polluted soils, sediments and groundwater, John Wiley \& Sons, 2009. 
[7] M.T. Alcantara, J. Gomez, M. Pazos, M.A. Sanroman, Electrokinetic remediation of PAH mixtures from kaolin, Journal of Hazardous Materials, 179 (2010) 1156-1160.

[8] M.T. Alcantara, J. Gomez, M. Pazos, M.A. Sanroman, Electrokinetic remediation of lead and phenanthrene polluted soils, Geoderma, 173 (2012) 128-133.

[9] A.B. Ribeiro, J.M. Rodriguez-Maroto, E.P. Mateus, H. Gomes, Removal of organic contaminants from soils by an electrokinetic process: the case of atrazine. Experimental and modeling, Chemosphere, 59 (2005) 1229-1239.

[10] A.B. Ribeiro, E.P. Mateus, J.-M. Rodriguez-Maroto, Removal of organic contaminants from soils by an electrokinetic process: The case of molinate and bentazone. Experimental and modeling, Separation and Purification Technology, 79 (2011) 193-203.

[11] C. Risco, S. Rodrigo, R.L. Vizcaíno, A. Yustres, C. Saez, P. Cañizares, V. Navarro, M. Rodrigo, Removal of oxyfluorfen from spiked soils using electrokinetic soil flushing with linear rows of electrodes, Chemical Engineering Journal, 294 (2016) 65-72.

[12] C. Risco, H. Rubi-Juarez, S. Rodrigo, R.L. Vizcaíno, C. Saez, P. Canizares, C. Barrera-Diaz, V. Navarro, M. Rodrigo, Removal of oxyfluorfen from spiked soils using electrokinetic fences, Separation and Purification Technology, 167 (2016) 55-62.

[13] L.Y. Wick, L. Shi, H. Harms, Electro-bioremediation of hydrophobic organic soilcontaminants: A review of fundamental interactions, Electrochimica Acta, 52 (2007) 34413448.

[14] J.T. Cookson, Bioremediation Engineering: Design and Application, McGraw-Hill, New York, USA, 1995.

[15] M. Gavrilescu, Fate of pesticides in the environment and its bioremediation, Engineering in Life Sciences, 5 (2005) 497-526.

[16] R. Gill, M.J. Harbottle, J. Smith, S. Thornton, Electrokinetic-enhanced bioremediation of organic contaminants: A review of processes and environmental applications, Chemosphere, 107 (2014) 31-42.

[17] E. Mena, C. Ruiz, J. Villaseñor, M.A. Rodrigo, P. Cañizares, Biological permeable reactive barriers coupled with electrokinetic soil flushing for the treatment of diesel-polluted clay soil, Journal of hazardous materials, 283 (2015) 131-139.

[18] A.T. Yeung, Y.-Y. Gu, A review on techniques to enhance electrochemical remediation of contaminated soils, Journal of hazardous materials, 195 (2011) 11-29.

[19] S. Barba, J. Villaseñor, M.A. Rodrigo, P. Cañizares, Effect of the polarity reversal frequency in the electrokinetic-biological remediation of oxyfluorfen polluted soil, Chemosphere, 177 (2017) 120-127.

[20] R. Lopez-Vizcaino, V. Navarro, M.J. Leon, C. Risco, M.A. Rodrigo, C. Saez, P. Canizares, Scale-up on electrokinetic remediation: Engineering and technological parameters, Journal of Hazardous Materials, 315 (2016) 135-143.

[21] R. Lopez-Vizcaino, C. Risco, J. Isidro, S. Rodrigo, C. Saez, P. Canizares, V. Navarro, M.A. Rodrigo, Scale-up of the electrokinetic fence technology for the removal of pesticides. Part I: Some notes about the transport of inorganic species, Chemosphere, 166 (2017) 540-548.

[22] R. Lopez-Vizcaino, C. Risco, J. Isidro, S. Rodrigo, C. Saez, P. Canizares, V. Navarro, M.A. Rodrigo, Scale-up of the electrokinetic fence technology for the removal of pesticides. Part II: Does size matter for removal of herbicides?, Chemosphere, 166 (2017) 549-555.

[23] S.A. International, ASTM D2487. Classification of Soils for Engineering Purposes (Unified Soil Classification System), in, West Conshohocken, Pensilvania, USA., 2006.

[24] S.A. International, ASTM D4318. Standard Test Methods for Liquid Limit, Plastic Limit, and Plasticity Index of Soils, in, West Conshohocken, Pensilvania, USA., 2010.

[25] R. López-Vizcaíno, V. Navarro, M.J. León, C. Risco, M.A. Rodrigo, C. Sáez, P. Cañizares, Scale-up on electrokinetic remediation: Engineering and technological parameters, J. Hazard. Mater., 315 (2016) 135-143. 
[26] R. López-Vizcaíno, C. Risco, J. Isidro, S. Rodrigo, C. Saez, P. Cañizares, V. Navarro, M.A. Rodrigo, Scale-up of the electrokinetic fence technology for the removal of pesticides. Part I: Some notes about the transport of inorganic species, Chemosphere, 166 (2017) 540-548.

[27] R. López-Vizcaíno, C. Risco, J. Isidro, S. Rodrigo, C. Saez, P. Cañizares, V. Navarro, M.A. Rodrigo, Scale-up of the electrokinetic fence technology for the removal of pesticides. Part II: Does size matter for removal of herbicides?, Chemosphere, 166 (2017) 549-555.

[28] C. Risco, R. López-Vizcaíno, C. Sáez, A. Yustres, P. Cañizares, V. Navarro, M.A. Rodrigo, Remediation of soils polluted with 2,4-D by electrokinetic soil flushing with facing rows of electrodes: A case study in a pilot plant, Chem. Eng. J., 285 (2016) 128-136.

[29] C. Risco, S. Rodrigo, R. López Vizcaíno, A. Yustres, C. Saez, P. Cañizares, V. Navarro, M.A. Rodrigo, Removal of oxyfluorfen from spiked soils using electrokinetic soil flushing with linear rows of electrodes, Chem. Eng. J., 294 (2016) 65-72.

[30] C. Risco, S. Rodrigo, R. López-Vizcaíno, C. Sáez, P. Cañizares, V. Navarro, M.A. Rodrigo, Electrokinetic flushing with surrounding electrode arrangements for the remediation of soils that are polluted with 2,4-D: A case study in a pilot plant, Sci. Total Environ., 545-546 (2016) 256-265.

[31] C. Risco, S. Rodrigo, R. López-Vizcaíno, A. Yustres, C. Sáez, P. Cañizares, V. Navarro, M.A. Rodrigo, Electrochemically assisted fences for the electroremediation of soils polluted with 2,4-D: A case study in a pilot plant, Separation and Purification Technology, 156, Part 2 (2015) 234-241.

[32] C. Risco, H. Rubí-Juárez, S. Rodrigo, R. López Vizcaíno, C. Saez, P. Cañizares, C. BarreraDíaz, V. Navarro, M.A. Rodrigo, Removal of oxyfluorfen from spiked soils using electrokinetic fences, Separation and Purification Technology, 167 (2016) 55-62.

[33] C. Risco, H. Rubí-Juárez, S. Rodrigo, R. López-Vizcaíno, C. Saez, P. Cañizares, C. BarreraDíaz, V. Navarro, M.A. Rodrigo, Removal of oxyfluorfen from spiked soils using electrokinetic soil flushing with the surrounding arrangements of electrodes, Sci. Total Environ., 559 (2016) 94-102.

[34] S.A. International, ASTM D7263. Standard Test Methods for Laboratory Determination of Density (Unit Weight) of Soil Specimens, in, West Conshohocken, Pensilvania, USA., 2009.

[35] S.A. International, ASTM D2216. Standard Test Methods for Laboratory Determination of Water (Moisture) Content of Soil and Rock by Mass, in, West Conshohocken, Pensilvania, USA., 2010.

[36] E. Mena, J. Villaseñor, P. Cañizares, M. Rodrigo, Influence of electric field on the remediation of polluted soil using a biobarrier assisted electro-bioremediation process, Electrochimica Acta, 190 (2016) 294-304. 